\title{
Imaging Low Z Materials in Crystalline Environments Via Scanning Transmission Electron Microscopy
}

\author{
SD Findlay $^{1 *}$, N Shibata ${ }^{2}$, Y Ikuhara ${ }^{2}$, L Clark $^{3}$, HG Brown ${ }^{4}$, TC Petersen ${ }^{1}$, DM Paganin ${ }^{1}$ and \\ MJ Morgan ${ }^{1}$ \\ 1. School of Physics and Astronomy, Monash University, Melbourne, Australia \\ 2. Institute of Engineering Innovation, The University of Tokyo, Tokyo, Japan \\ 3. School of Physics and Astronomy, University of Glasgow, Glasgow, UK \\ 4. National Centre for Electron Microscopy, Lawrence Berkeley National Laboratory, Berkeley, USA \\ * Corresponding author: scott.findlay@monash.edu
}

Amongst atomic-resolution scanning transmission electron microscopy (STEM) imaging modes, annular dark-field (ADF; shown schematically in Fig. 1(a)) is particularly popular since it produces directly interpretable images - with bright peaks indicating atomic columns - over a wide thickness range and is fairly robust against aberrations. The thickness-defocus tableaus of ADF images in Fig. 1(b) for $\mathrm{SrTiO}_{3}$ and Fig. 1(c) for $\mathrm{Al}_{3} \mathrm{Li}$ are consistent with this. That $\mathrm{ADF}$ peak intensities scale roughly as the atomic number $(\mathrm{Z})$ squared helps distinguish elements but also means low $\mathrm{Z}$ element columns may not be visible when high $\mathrm{Z}$ element columns are nearby - the $\mathrm{O}$ columns in Fig. 1(b) and Li columns in Fig. 1(c) are not visible - necessitating other methods to identify low Z element columns. This talk with review some atomic-resolution STEM imaging modes better suited to observing low Z element columns.

A decade ago it was found that so-called annular bright field (ABF) imaging, which uses an annular detector spanning the outer portion of the bright field disk, also produces directly interpretable images with dark troughs indicating atomic columns - over a wide thickness range [1,2]. Moreover, the atomic number dependence is relatively weak and so both low and high $\mathrm{Z}$ element columns are visible simultaneously. The thickness-defocus tableaus of ABF images in Figs. 1(b) and (c) are consistent with this. $\mathrm{ABF}$ has since found many applications, including probing oxygen octahedra rotation in perovskites and charging/discharging in lithium battery materials. Further improvements have been suggested, such as so-called enhanced ABF (eABF) imaging [3] shown in the thickness-defocus tableaus in Figs. 1(b) and (c), and also more case-specific optimisations [4]. We overview some guiding principles and limitations of $\mathrm{ABF}$ imaging and its variants for imaging low $\mathrm{Z}$ elements.

Recent developments in fast-readout pixel array detectors allow not only more scope for synthesizing optimal annular detector configurations [4] but also for new imaging modes. In particular, differential phase contrast (DPC) [5,6] and ptychography [7-9] methods allow good imaging of low Z element columns and are dose-efficient [6,8]. The thickness-defocus tableaus of phase reconstructions integrated DPC (iDPC) images in the parlance of Ref. [6] - in Figs. 1(b) and (c) are consistent with this. DPC and ptychography yield quantitative reconstructions of the projected electrostatic potential when the phase object approximation holds, but this breaks down for crystals thicker than a few nanometers when using atomically-fine electron probes $[10,11]$. The thickness-defocus tableaus of iDPC images in Fig. 1(b) and (c) shows this breakdown once it is appreciated that the true projected electrostatic potential should increase linearly with thickness. Specifically, in the iDPC images around zero relative defocus (relative to the sample midplane, the conditions seemingly most favourable for DPC STEM [10]) the iDPC signal only increases for the first 50-100 $\AA$. We overview some approaches and limitations to DPC imaging and other phase contrast imaging modes for imaging low $\mathrm{Z}$ elements [12]. 
References:

[1] E Okunishi et al., Microsc Microanal 15(Suppl 2) (2009), p. 164.

[2] SD Findlay et al., Ultramicroscopy 110 (2010), p. 903.

[3] SD Findlay et al., Ultramicroscopy 136 (2014), p. 31.

[4] J Gonnissen et al., Applied Physics Letters 105 (2014), p. 063116.

[5] K Müller et al., Nature Communications 5 (2014), p. 5653.

[6] I Lazić, EGT Bosch, S Lazar, Ultramicroscopy 160 (2016,) p. 265.

[7] CT Putkunz et al., Physical Review Letters 108 (2012), p. 073901.

[8] H Yang et al., Nature Communications 7 (2016), p. 12532.

[9] Y Jiang et al., Nature 559 (2018), p. 343.

[10] R Close et al., Ultramicroscopy 159 (2015,) p. 124.

[11] K Müller-Caspary et al., Ultramicroscopy 178 (2017), p. 62.

[12] This research was supported under the Australian Research Council Discovery Projects funding scheme (Project No. DP160102338). We thank all our collaborators who have contributed so much to the various pieces of research overviewed in this talk.

(a)

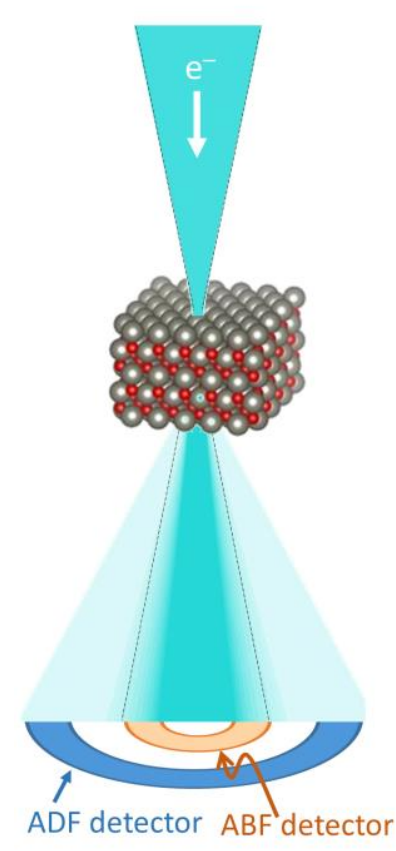

(b)
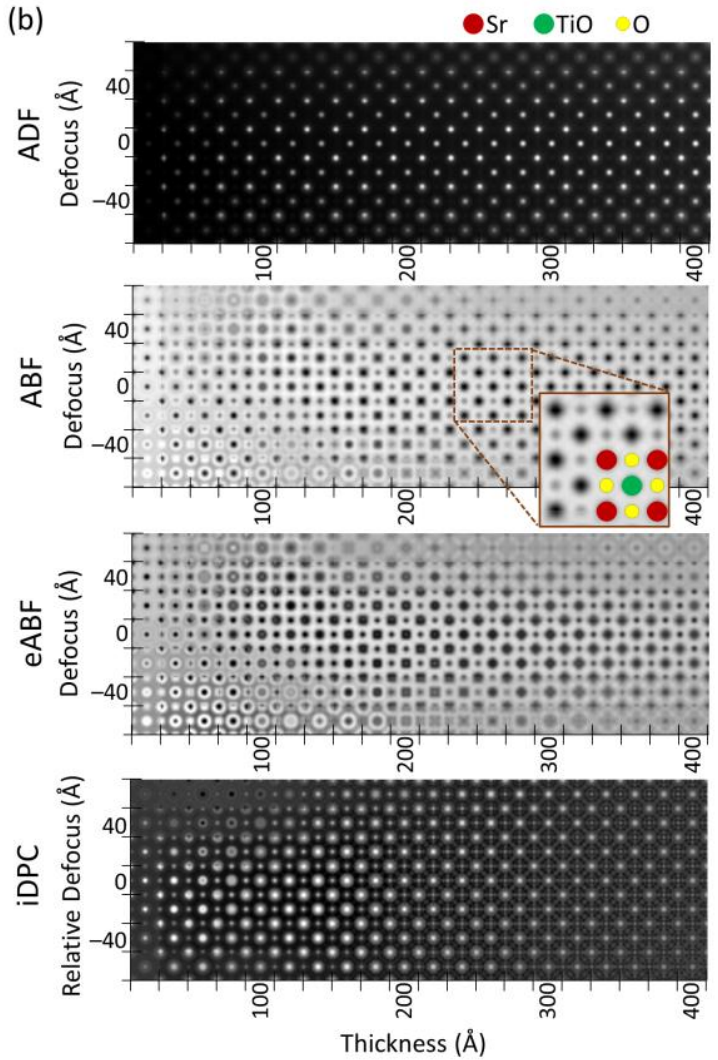

(c)
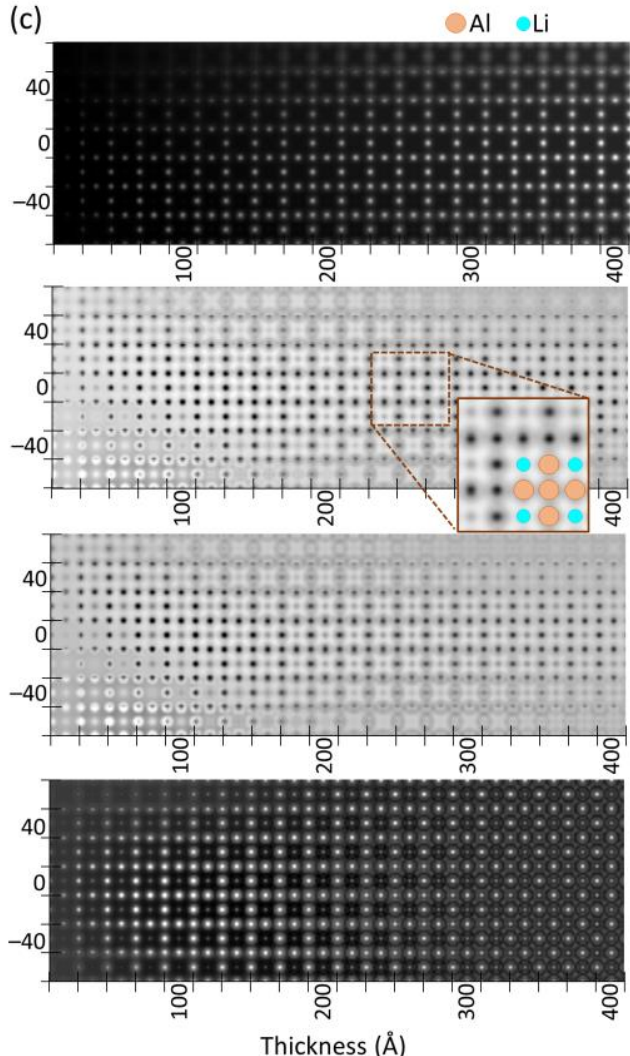

Figure 1. (a) Schematic of STEM experiment showing two annular detectors (for ADF and ABF) in the diffraction plane. (b) Thickness-defocus tableaus for ADF, ABF, eABF and iDPC of $\mathrm{SrTiO}_{3}$ [001] simulated using an absorptive model. Accelerating voltage $=200 \mathrm{kV}$, probe-forming aperture semiangle $=23 \mathrm{mrad}$, HAADF detector range $=81-228 \mathrm{mrad}, \mathrm{ABF}$ detector range $=11.5-23 \mathrm{mrad}$. Whereas the $\mathrm{ADF}, \mathrm{ABF}$ and $\mathrm{ABF}$ tableaus are given with defocus relative to the sample entrance surface, in the case of iDPC the defocus is given relative to the specimen mid-plane. (c) As for (b) but for $\mathrm{Al}_{3} \mathrm{Li}$ [001]. 